\title{
La noción de educación de calidad a nivel superior: una reflexión desde el discurso del desarrollo
}

\author{
Clara Zafra, Miguel Ángel \\ Universidad Veracruzana, México \\ mclara@uv.mx
}

\author{
Vega Zárate, César \\ Universidad Veracruzana, México \\ cevega@uv.mx
}

Resumen - En esta investigación se presenta una reflexión acerca de la noción de educación de calidad en la educación superior desde las teorías que constituyen el discurso del desarrollo. Este preámbulo es la pauta que sirve para abordar la importancia de la educación superior para el desarrollo de los países, y como la acreditación juega un papel protagónico del mismo discurso. Además, se reflexiona la educación de calidad desde los Objetivos de Desarrollo Sostenible de la agenda 2030.

Palabras clave - Educación de calidad; Educación superior; Discurso del desarrollo;

\begin{abstract}
This research presents a review reflection on quality Education in Higher Education. From the theories that constitute the Development Discourse. This preamble is the guideline that serves to address the importance of higher education for the development of countries. so how accreditation plays a leading role in the same discourse. Further, quality education is reflected from the Sustainable Development Goals of the 2030 agenda.
\end{abstract}

Keywords - Quality education; Higher education; Development discourse;

Interconectando Saberes, 2020

ISSN: 2448-8704

\section{INTRODUCCIÓN}

En los últimos tres decenios la educación superior se ha visto inmersa en una serie de cambios estructurales; desde el proceso de enseñanza-aprendizaje hasta en su gestión institucional. Con la llegada del discurso del desarrollo en el siglo XX la educación se convirtió también parte del discurso, y desde entonces es pieza fundamental en las agendas de desarrollo de los países para reducir la desigualdad y la pauperización.

En una primera aproximación, la educación y la adquisición de conocimientos forman parte de un proceso de apropiamiento de valores, actitudes, creencias y hábitos del ser humano para su desarrollo, y así este pueda enfrentar los retos de la vida. Luengo (2004) menciona que la educación es un fenómeno que nos concierne a todos desde que nacemos y es parte fundamental para alcanzar un nivel de calidad de vida plena.

Fecha de Recepción: 19 de junio de 2020

Fecha de Aceptación: 22 de julio de 2020 Fecha de Publicación: 24 de julio de 2020 
La educación superior es necesaria para el desarrollo de los países, ya que en este nivel se forma el capital humano que se requiere para enfrentar los problemas de orden global. Así es como en las últimas décadas, diversas investigaciones de gran importancia han surgido (Moreno y Ruiz, 2010), atribuyéndole a la educación superior un papel importante en el desarrollo económico y social de los países.

De acuerdo con Reyes la definición general de desarrollo incluye "la especificación de que los grupos sociales tienen acceso a organizaciones y servicios básicos de educación, salud y nutrición" (2001, p11). Es posible indicar que el desarrollo considerado una condición social de un país guarda una relación estrecha con la educación, ya que la educación potencializa el capital humano que requiere un país para progresar y obtener niveles óptimos de desarrollo.

Por su parte la Organización de las Naciones Unidas para la Educación (2019) añade que la educación de calidad debe ser concebida como el motor central que garantizará el desarrollo sostenible en los países. Por ello, esta investigación retoma el discurso del desarrollo, que de acuerdo con Escobar (2007) y Esteva (1996) surge cuando el presidente de los Estados Unidos en 1949 Harry Truman anunció al mundo el concepto de "trato justo", para resolver problemas de las áreas subdesarrolladas. Dentro del discurso se hacía alusión a términos como: miseria, economía primitiva, industrialización, urbanización, crecimiento de la producción, calidad de vida y adopción de valores culturales modernos. Estos términos estaban y están estrechamente relacionados con la educación y el conocimiento de las personas para el progreso.

En este sentido, el principal objetivo del presente artículo es contribuir al conocimiento desde tres aspectos principales: primero, realizar una revisión teórica de las principales teorías del desarrollo que conforman el discurso del desarrollo y cómo estás tienen una relación con la educación de calidad. En segundo lugar, reflexionar sobre la educación de calidad en la educación superior a partir de los Objetivos de Desarrollo Sostenible (ODS) de la agenda 2030; y tercero señalar cómo la acreditación de la educación superior contribuye al desarrollo de los países.

La investigación se fundamenta en la revisión de literatura con respecto a los conceptos de desarrollo y educación, y desde una postura crítica se reflexiona sobre la relación estrecha que guardan ambos conceptos.

\section{EL DISCURSO DEL DESARROLLO}

Con la llegada del discurso del desarrollo, la humanidad tuvo que adaptarse a una nueva clasificación: países en desarrollo y subdesarrollo, haciendo notar de una forma más explícita las desigualdades y la pauperización en el mundo. Escobar (2007) atribuye lo anterior, como la invención del tercer mundo. En enero de 1949 cuando el presidente de los Estados Unidos, Harry 
Truman ${ }^{1}$ tomó posesión, anunció al mundo el discurso del desarrollo "trato justo" para resolver los problemas de las áreas subdesarrolladas del globo:

Más de la mitad de la población del mundo vive en condiciones cercanas a la miseria. Su alimentación es inadecuada, es víctima de la enfermedad. Su vida económica es primitiva y está estancada. Su pobreza constituye un obstáculo y una amenaza tanto para ellos como para las áreas más prósperas. Por primera vez en la historia, la humanidad posee el conocimiento y la capacidad para aliviar el sufrimiento de estas gentes... Creo que deberíamos poner a disposición de los amantes de la paz los beneficios de nuestro acervo de conocimiento técnico para ayudarlos a lograr sus aspiraciones de una vida mejor... Lo que tenemos en mente es un programa de desarrollo basado en los conceptos del trato justo y democrático... Producir más es la clave para la paz y la prosperidad. Y la clave para producir más es una aplicación mayor y más vigorosa del conocimiento técnico y científico moderno (Truman, 1967, tomado de Escobar, 2007, p. 19).

Con lo anterior, aparece el discurso del desarrollo que no solo era exclusivo Para los Estados Unidos, sino se adaptaba históricamente con la finalización de la segunda guerra mundial, y donde el mundo necesitaba una estrategia para activar la economía y otros problemas de orden social. De acuerdo con
Escobar (2007) dentro de este discurso se hacía alusión a términos como: miseria, economía primitiva, industrialización, urbanización, crecimiento de la producción, calidad de vida y adopción de valores culturales modernos.

En este sentido, aunado a lo anterior, hay que destacar que la educación se encuentra inmersa dentro de los problemas del tercer mundo. Arnove (2006) señala que la implementación de la política estructural para la liberalizar las economías y el suministro de servicios sociales básicos han impedido que la población que vive en países subdesarrollados tengan acceso a una educación de calidad e igualdad de oportunidades en la vida. En efecto, la educación toma un papel importante en el progreso de un país que coadyuvará al bienestar social y económico.

Entonces, derivado de lo anterior, surge la interrogante: ¿Existe alguna relación entre la educación y el discurso del desarrollo?, la respuesta es evidente, sí. El desarrollo construido como un discurso político donde los Estados Unidos toma un protagonismo de querer tener un "trato justo" con los menos desarrollados, la educación como un problema común en los países pobres, cobra una gran relevancia sobre los problemas que hay que atender y orientar. 
De ahí que en las principales agendas de desarrollo establezcan objetivos para alcanzar y garantizar la mejora en la educación en el mundo en todos los niveles, y de esta manera se contribuya a lograr el progreso social y económico.

Reyes (2001) define al desarrollo como una "condición social dentro de un país, en la cual las necesidades auténticas de su población se satisfacen con el uso racional y sostenible de recursos y sistemas naturales, además incluye que los grupos sociales posean acceso a organizaciones y a servicios básicos como educación, vivienda, salud, nutrición, y, sobre todo, que sus culturas y tradiciones sean respetadas dentro del marco social de un estado-nación en particular" (p.1).

Teniendo en cuenta esta concepción del desarrollo, cabe destacar que las teorías del desarrollo se fundamentan en la identificación de estructuras socioeconómicas y económicas para el progreso de un país. Desde hace ocho décadas han surgido diferentes modelos de desarrollo con el propósito de minimizar los problemas del tercer mundo, sin embargo, en la actualidad siguen persistiendo dichos problemas.

Por lo que comprende a la educación, en cada modelo se le ha considerado como eje modulador para que la población obtenga herramientas y conocimiento para su desarrollo personal, y en conjunto contribuyan al desarrollo de su nación. En la figura 1, se observa una línea del tiempo acerca de las teorías del desarrollo, y en donde aparece la educación como objetivo del discurso del desarrollo. Figura 1. Línea del tiempo de los momentos
importantes en relación con las teorías del desarrollo.

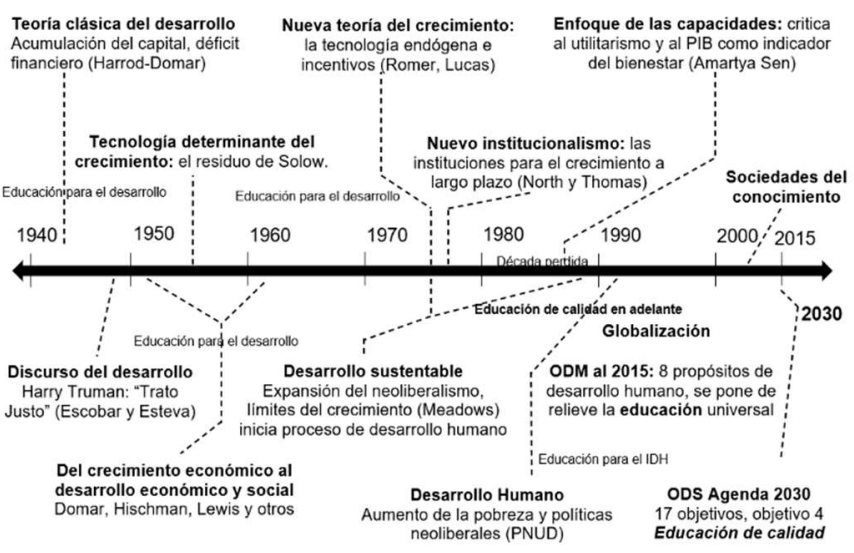

Fuente: Elaboración propia con información obtenida de Oriol Prats (2006), Cuéllar y Moreno (2009), y de ODM y ODS de la Agenda 2030.

En tal sentido, la idea de desarrollo se ha modificado con el paso del tiempo sin perder su esencia; apoyar a los menos adelantado, en tanto la educación ha sido parte del discurso como motor del desarrollo. Sin embargo, durante este lapso no se ha logrado erradicar la pobreza, desigualdad y el hambre en el mundo, al contrario, pareciera que más personas en la actualidad están padeciendo estos problemas del subdesarrollo, que les impide optar por una mejor calidad de vida en su desarrollo personal.

Aunque no se pueda observar a simple vista, la educación en el mundo ha sido un gran reto que han tenido que enfrentar los países subdesarrollados. Por tanto, la educación juega un rol protagónico en el tema desarrollista, ya que, 
aunque los países se han preocupado por mejorar los sistemas educativos, persisten problemas de fondo. En efecto, han surgido reformas $y$ reestructuraciones con el afán de mejorar la educación y no se ha logrado alcanzar los estándares de una educación de calidad, y menos reducir la pauperización y la desigualdad social.

En consecuencia, de que todas las estrategias para mejorar la educación no han funcionado, y con la llegada de la globalización, surge un nuevo paradigma: la "educación de calidad". Que dentro del marco de las teorías del desarrollo surge precisamente durante la década pérdida ${ }^{2}$, y es cuando inicia a tomar un lugar importante en la agenda política de educación, en el contexto latinoamericano fungió como pieza clave que ha fundamentado las reformas educativas y modelos a seguir para construir un nivel educativo sólido (Orozco, Olaya, y Villate, 2009).

En este sentido, desde la década de 1980 se instaura el paradigma de la educación de calidad y se adopta en las principales agendas del discurso del desarrollo. En el marco de las alternativas al desarrollo, surge el Programa de las Naciones Unidas para el Desarrollo (PNUD) en 1967. Sin embargo, fue hasta 1990 cuando se lanza el llamado paradigma de "desarrollo humano", como teoría o propuesta destinada a sustituir el concepto tradicional de desarrollo identificado como crecimiento económico y medido principalmente por el nivel de ingreso per cápita (Tünnermann,

${ }^{2}$ La década pérdida en América Latina se refiere a la crisis económica que detuvieron el desarrollo en 1980.
2003). Este paradigma de desarrollo humano posee una estrecha relación con la educación de calidad, ya que se enfatiza en centrar la educación como el motor para generar las capacidades y oportunidades de las personas, inclusive la educación forma parte del Índice de Desarrollo Humano (IDH).

Amartya Sen es el principal exponente del desarrollo humano, cuyas aportaciones al desarrollo son relevantes, y su tesis se centra en la crítica utilitarista, acerca de dejar de medir el desarrollo por el ingreso per cápita y hacerlo por el $\mathrm{IDH}$, centrado en la medición de "tres elementos esenciales de la vida humana: Iongevidad, conocimientos y niveles decentes de vida" (Bedoya, 2011, p.264). Desde otras palabras, Tellería (2015) menciona que dicho índice mide los niveles de salud, educación e ingresos de la población de cada país en el mundo.

Conviene subrayar que el desarrollo humano tiene definida la educación como motor importante para la vida de las personas. Pero ¿qué pasa realmente con la educación en el mundo?, la respuesta sigue siendo la misma, persisten grandes brechas entre los países. Aunque la matricula en el mundo ha incrementado exponencialmente continúan los retos de garantizar una educación de calidad.

Seguir viendo el desarrollo a través del IDH y Producto Interno Bruto (PIB) en los países, provocan que las brechas del ingreso crezcan y se 
incremente la desigualdad (Pérez-Najera, 2020). En el contexto de la educación estos indices también generan un efecto que se ve reflejado en la desiguadad de la educación, un ejemplo claro sería el nivel educativo de los Estados Unidos comparado con el de México, país del tercer mundo. En tal sentido, la brecha sigue siento inmensa, por ello es necesario establecer estrategias en los países que garanticen una educación de calidad.

En concreto, el discurso del desarrollo a través de cada una de las teorías del desarrollo ha tenido una relación estrecha con los temas relacionados con la educación, dado que en cada teoría siempre se ha discutido la importancia de la educación para el desarrollo de los países. Inclusive la llegada de la globalización y el paradigma de la educación de calidad han establecido una mayor atención en los organismos internacionales para establecer objetivos de "educación universal", "igualdad educativa" y "educación de calidad".

\section{LA EDUCACIÓN DE CALIDAD Y LOS} OBjetivos dEL DESARROLLO SOSTENIBLE

El desarrollo considerado como una condición social de un país (Reyes, 2001), guarda una relación estrecha con la educación. Villalobos y Pedroza sostienen que "la educación es un elemento fundamental en la formación de capital humano, y, por tanto, propicia el crecimiento económico, y desarrollo de un país" (2009, p.303). En este sentido, la educación es un elemento clave que potencializa el desarrollo. Sin embargo, con la llegada de la globalización la educación ha tenido que adoptar la calidad (Rivas, 2014) como mecanismo moderno de mejora, propiciando así la llegada del discurso de la educación de calidad qué contribuirá al progreso.

La educación de calidad se ha convertido en el principal objetivo perseguido por las instituciones educativas para la contribución al desarrollo humano de los países, por ello figura en las principales agendas de desarrollo de instituciones internacionales como la Organización de las Naciones Unidas (ONU) y el Fondo de las Naciones Unidas para la Infancia (UNICEF). Desde nuestro enfoque, retomaremos la definición de educación de calidad, entendida como la mejora de los procesos educativos y pedagógicos que potencializa el desarrollo humano, además de remitirse a consideraciones éticas y objetivos propios de la educación que involucra aspectos económicos, políticos y sociales para el desarrollo de los países, (Orozco et al., 2009; Lemaitre, Maturana, Zenteno, y Alvarado, 2012; Avendaño, Paz, y Parada, 2016).

Del discurso del desarrollo económico y social al discurso de la educación de calidad han surgido varias propuestas y objetivos para promover una educación eficiente que provea herramientas para el desarrollo humano de las personas. En el año 2000 la ONU derivado de las iniciativas del discurso del desarrollo contemporáneo, establece los Objetivos de Desarrollo del Milenio (ODM), los cuales consistían en ocho grandes principios: "(1) erradicar la pobreza extrema y el hambre, (2) enseñanza primaria universal, (3) igual de género y 
empoderamiento de la mujer, (4) reducir la mortalidad de niños, (5) salud materna, (6) combatir el VIH/sida, (7) garantizar la sostenibilidad del medio ambiente, y (8) fomentar la alianza mundial para el desarrollo" (Programa de las Naciones Unidas para el Desarrollo, 2015, p.4-8).

Los ODM son "un instrumento que permite unir esfuerzos en un mundo globalizado que exige respuestas de igual naturaleza, siendo una referencia para las políticas y acciones que numerosas organizaciones oficiales y no oficiales implementan en la actualidad" (González, 2011, p.34). Los objetivos son cruciales para la estructura de directrices en las agendas de desarrollo, en el contexto de la educación se convierten en un gran reto de alcanzar, en especial en los países subdesarrollados donde las desigualdades económicas y sociales impiden que la población tenga el acceso básico a la educación elemental.

Tellería (2015) menciona de acuerdo con la Declaración del Milenio de las Naciones Unidas, qué el año 2015 sería el plazo en el cual los objetivos del milenio serían alcanzados en su totalidad. Sin embargo, en la realidad no fue así, de hecho, desde el 2013 las Naciones Unidas estaban replanteando la agenda de desarrollo posterior, qué daría el seguimiento a estos objetivos. En materia de educación durante estos quince años se lograron grandes avances, por ejemplo: la educación básica intensifico el número de matrícula, se construyeron escuelas dignas especialmente en países subdesarrollados, y se constituyeron políticas públicas en diferentes países con el objetivo de garantizar la educación primaria.

De acuerdo con el informe del PNUD (2015) las estrategias basadas en los objetivos del milenio han logrado generar un efecto en la educación. En el 2000 no asistían cerca de 100 millones de niños a las escuelas, cifra que logro reducirse a 57 millones en el año 2015. Estos resultados representan un gran avance para el conocimiento, sin embargo, desde la perspectiva del discurso del desarrollo no ha sido suficiente para disminuir las desigualdades económicas y sociales. Por ello, las Naciones Unidas han replanteado los ODM a los ODS.

Cabe señalar que desde 1980, el discurso de la educación de calidad se encuentra incluido como objetivo en las agendas de desarrollo, y se ha visto reflejada en la formulación de políticas públicas para atender esta necesidad de la sociedad (Orozco et al., 2009). Los ODM significaron el inicio de un gran reto para los países, en especial para los subdesarrollados. En el 2015 después no alcanzar los ODM, en la cumbre de la Naciones Unidas en New York el 25 de septiembre, se dan a conocer y se aprueban los ODS en el documento "Transformar Nuestro Mundo: la Agenda 2030 para el Desarrollo Sostenible". En dicha agenda se incluyen diecisiete objetivos, donde se establece nuevamente "poner fin a la pobreza, luchar contra la desigualdad y la injusticia, y hacer frente al cambio climático sin que nadie quede atrás para el 2030" (Organización de las Naciones Unidas, 2015). 
Dentro de los ODS se enfatiza en la cooperación, promoción de la inclusión y equidad en un marco de derechos para todos y todas. En efecto las Naciones Unidas le dan continuidad al discurso del desarrollo, haciendo notar que los problemas de pobreza y desigualdad siguen persistiendo, y por ello se pone de meta la agenda 2030. Con relación al tema de la educación se reformula el objetivo de educación universal, hacía una "educación de calidad" en todos los niveles educativos para todos.

La educación de calidad será el motor central para garantizar el desarrollo sostenible en los países (Organización de las Naciones Unidas para la Educación, 2019). En contraste con los ODM, se enfatiza en la educación primaria y secundaria, la inclusión y equidad, y la promoción de aprendizaje durante toda la vida para todos. De acuerdo con la Organización de las Naciones Unidas para la Educación, la Ciencia y la Cultura (UNESCO) en esta agenda se hace énfasis en la educación y se establece como objetivo elemental para el desarrollo: "garantizar una educación inclusiva y equitativa de calidad y promover oportunidades de aprendizaje a lo largo de la vida para todos" (Organización de las Naciones Unidas para la Educación, 2019, p.5).

Este objetivo de educación de calidad se desglosa en siete metas y tres medios de implementación. Cada meta tiene una relación estrecha para apoyar otros objetivos de la misma agenda 2030 (ver Figura 2), por esta razón, se considera la educación como el motor para el desarrollo sostenible que coadyuvará al "bienestar y salud, igualdad de género, trabajo decente y crecimiento económico, producción y consumo responsable, y la acción por el clima" (Organización de las Naciones Unidas para la Educación, 2019, p.7).

Figura 2. Relación del objetivo de educación de calidad con otros objetivos de la agenda 2030.

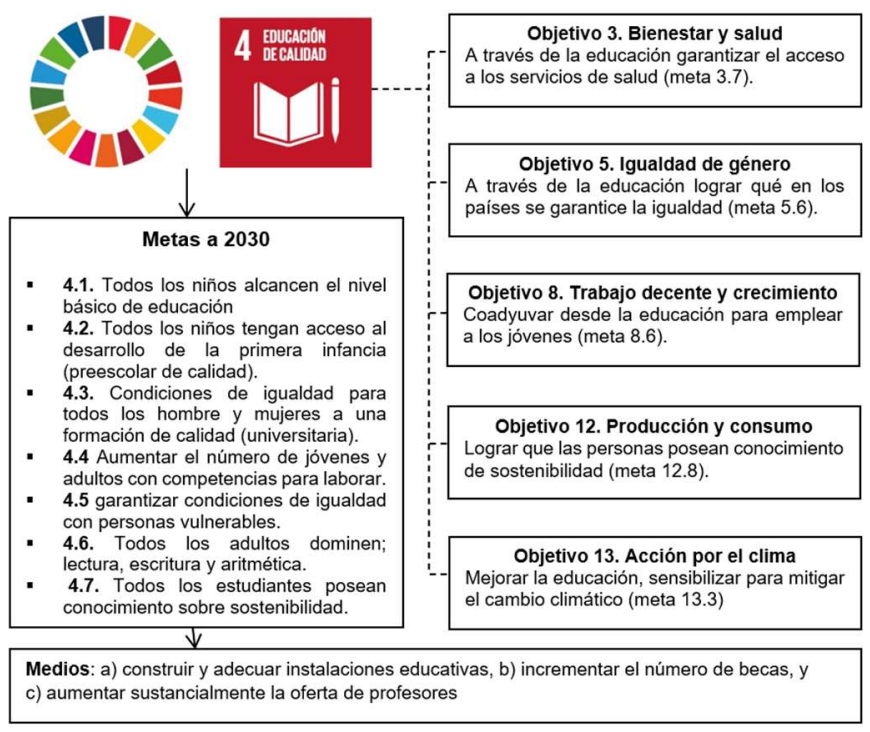

Fuente: Elaboración propia con información del documento de la Organización de las Naciones Unidas para la Educación (2019) sobre los ODS de la agenda 2030. Nota: se sintetizó la información del documento de una forma clara y precisa.

Es entonces que, desde esta postura, la educación de calidad se convierte en un objetivo esencial que influye de formas directa e indirecta con otros objetivos para alcanzar un desarrollo sostenible en el mundo. Cabe señalar que estas metas son ambiciosas de alcanzar para el año 2030, sin embargo, diferentes instituciones alineadas a las Naciones Unidas han establecidos estrategias para hacer llegar estos objetivos a todos los países sin excepciones, en especial aquellos que se encuentran en desarrollo o subdesarrollo. 
En concreto, los ODS establecen que la educación de calidad es el motor para el desarrollo sostenible, sin embargo, es imprescindible reflexionar sobre los retos que deben de asumir las instituciones educativas para el logro de este objetivo, en especial la educación superior, donde persisten diversas problemáticas.

De acuerdo con el objetivo 4.3 de la agenda 2030 de los ODS, la educación superior debe lograr al 2030: "asegurar el acceso en condiciones de igualdad para todos los hombres y las mujeres a una formación técnica, profesional y superior de calidad, incluida la enseñanza universitaria" (Organización de las Naciones Unidas para la Educación, 2019, p. 12).

Definitivamente es un gran reto para la educación superior lograr la igualdad y sobre todo la oferta de programas de calidad. Desde la propia concepción de la educación de calidad las Instituciones de Educación Superior (IES) deben enfrentar distintos retos dentro de los cuales destacan: lograr instalaciones dignas, educación inclusiva, igualdad de género e inclusión, acceso a las tecnologías de la información y la comunicación, docentes eficaces, la generación y aplicación del conocimiento.

En este sentido, Silas sostiene de acuerdo con la Declaración Mundial sobre la Educación Superior del Siglo XXI de la UNESCO; que la calidad en la educación superior debería mejorar y comprender todas las funciones y actividades: enseñanza y programas académicos, investigación y becas, personal, estudiantes, edificios, instalaciones, equipamientos y servicios a la comunidad y al mundo universitario $(2014$, p.2). En efecto la educación superior tendrá que impulsar el proceso de modernización y adaptarse a los procesos de rendición de cuenta de la política educativa a través de la evaluación, certificación y acreditación.

\section{LA EDUCACIÓN SUPERIOR Y LA ACREDITACIÓN}

La educación superior es considerada la última fase del proceso de aprendizaje académico, fase donde el estudiante se especializa en alguna área del conocimiento afín a sus gustos y capacidades para en el corto plazo ingresar al mercado laboral. La educación superior es el principal motor del desarrollo económico en los países, para ello los gobiernos deben de establecer estrategias innovadoras de (Moreno y Ruíz, 2010, p.11):

1. Infraestructura científica;

2. Oferta de personal altamente calificado en investigación; y

3. Una estrecha y funcional vinculación entre los centros de investigación y las empresas productivas.

Es decir, los gobiernos de los países que deseen lograr el desarrollo económico deben de esforzarse en mejorar el capital local, físico y humano, qué a través de la investigación puedan alcanzar el desarrollo, además de crear espacios y condiciones para asegurar una educación superior digna, y bajo la visión de educación de calidad establecida en los ODS de la agenda 2030. 
Desde el enfoque de educación de calidad, en el sector de la educación superior se refiere a la mejora de los servicios educativos que se le brinda a la sociedad, qué potencializa el desarrollo económico, social y humano. De acuerdo con Orozco y otros (2009) la educación de calidad está incluida en la agenda política desde 1980, y desde ese momento la educación superior ha interpretado este objetivo desde diferentes concepciones, en el caso de Latinoamérica las universidades han adoptado la evaluación, certificación y acreditación como medios para potencializar la educación de calidad que ofertan a la sociedad.

Egigio y Haug definen la acreditación como "aquel proceso voluntario por medio del cual una institución educativa se somete a la opinión de un organismo externo, con la intención de obtener un reconocimiento público de la calidad de su quehacer educativo" (2006, p.94). Así pues, la acreditación debe asumirse como un instrumento necesario para fortalecer y mejorar los procesos educativos.

Hernández (2006) sostiene que las IES buscan evaluarse, certificarse y acreditarse para mantenerse vigentes y actualizadas, y responden al resultado de la política internacional que pone de relieve reformar y realizar cambios en materia de educación de calidad. Aunado a ello, se han intensificado estos procesos por la influencia de organismos como: la UNESCO, Comisión Económica para América Latina y el Caribe (CEPAL), Organización de Estados
Iberoamericanos para la Educación, la Ciencia y la Cultura (OEI), Organización para la Cooperación y el Desarrollo Económicos (OCDE), Fondo Monetario Internacional (FMI), Banco Interamericano de Desarrollo (BID) y el Banco Mundial (BM). Estos organismos internacionales poseen concepciones distintas, sin embargo, ponen de acento que las IES deben adoptar una cultura de calidad, evaluación, y acreditación a través de estándares internacionales, y en efecto contribuyan a la rendición de cuentas y desarrollo de una región. Consecuentemente, la mejora de la educación superior contribuirá a resolver otros problemas establecidos en el discurso del desarrollo, tales como la pobreza, desigualdad, racismo, salud, entre otros.

En este sentido; la evaluación, certificación y acreditación son estrategias que han adoptado las IES para obtener reconocimiento, incrementar su demanda, contribuir a la rendición de cuentas, y en efecto al desarrollo de los países. Hernández (2006) menciona que los procesos de acreditación responden a la globalización, y mundialización, además de ser impulsados por organismos internacionales. De ahí qué, la acreditación de la educación superior sea también el resultado del discurso del desarrollo.

Tünnermann (2008) sostiene que el mejoramiento de la calidad y la integración de la educación superior son motores para la aceleración del desarrollo integral de la región de un país. Hoy en día la acreditación de la educación superior ha tomado mucha relevancia para los gobiernos, ya 
que responde a la rendición de cuentas y es detonante del desarrollo económico, social y humano. En la Figura 3, se indica de qué manera la acreditación contribuye al desarrollo de los países.

Figura 3. La acreditación de la educación superior y la contribución al desarrollo.

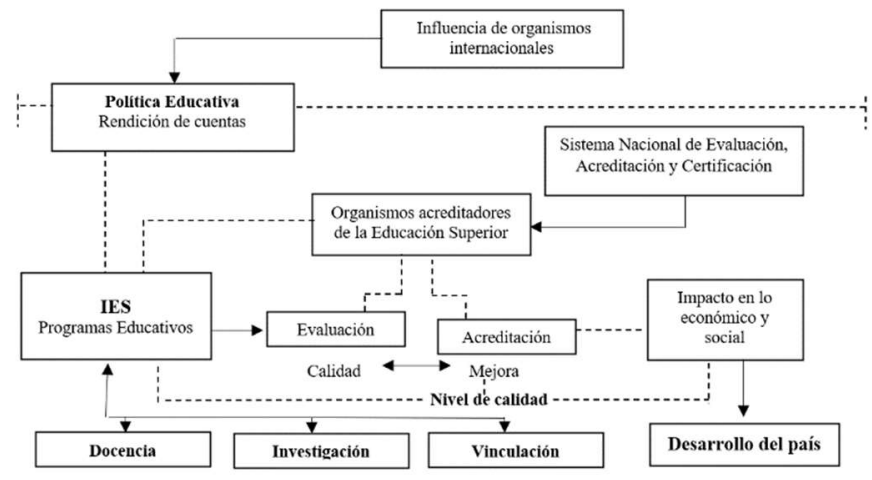

Fuente: elaboración propia con información de López y otros (2009) Egigio y Haug (2006); Mondragón (2006); Rodríguez (2007); González (2008); Claverie, González y Pérez (2008); y, Bernasconi y Rodríguez (2018).

En particular, la acreditación de la educación superior contribuye al desarrollo de un país, ya que fundamenta la mejora continua de la educación con el afán de lograr una educación de calidad. Munive (2007, p.397) sostiene que la acreditación no debe asumirse como la simple conquista o adjudicación de una constancia de un órgano o instancia acreditadora, sino en la integración formal de los procesos de gestión y funciones sustantivas de las universidades para lograr profesionales capaces de contribuir al desarrollo de un país.

En México la acreditación de la educación superior surge a través de la implementación de reformas hechas por el gobierno federal por medio de la Secretaría de Educación Pública (SEP). propósito principal de estas reformas radica en elevar la calidad de la educación y mejorar el sistema educativo (López V., 2019). En este sentido, se comprende que la acreditación de la educación superior surge en México a partir de reformas federales que se alinean a los objetivos de las Naciones Unidas para alcanzar el desarrollo del país.

Desde la década de 1990 surgen organismos acreditadores de la educación superior, con el afán de asegurar la calidad en este nivel educativo. El principal organismo fueron los Comités Interinstitucionales para la Evaluación de la Educación Superior (CIEES), y posteriormente el Consejo para la Acreditación de la Educación Superior (COPAES) el cual reúne aproximadamente a treinta consejos de acreditación en las diferentes áreas del conocimiento. Dichos organismos cuentan con la validez y la competencia para evaluar, y decidir si las IES cumplen con los elementos de educación de calidad (Acosta, 2014).

Lo anterior, responde a los objetivos de las IES, ya que en sus planes de desarrollo se encuentra el aseguramiento de calidad a través de la acreditación. Por ello, hoy en día las universidades deben de agregar a sus funciones sustantivas (docencia, investigación y vinculación), la acreditación con la finalidad de responder a la política educativa (rendición de cuentas). 
Cabe reflexionar sobre la contribución que la acreditación ejercer sobre el desarrollo del país. ¿Verdaderamente la acreditación de la educación superior contribuye al desarrollo?, es complejo responder a dicha pregunta. Sin embargo, es importante señalar que las IES deberían realizar este proceso de evaluación no solo por obtener un reconocimiento de calidad, sino por contribuir a ofrecer una mejor educación a la sociedad, y de esta manera sumar esfuerzos para disminuir los problemas mencionados en el discurso del desarrollo.

Finalmente, la educación de calidad es un tema que nos concierne a todos, como ya se mencionó es considerada como el motor del desarrollo. Por ello, las IES deben de asumir este reto como un beneficio en el largo plazo que coadyuvará en la formación de profesionistas de calidad, qué en el corto plazo dirigirán el rumbo del país y atenderán los principales problemas económicos y sociales.

\section{CONCLUSIONES}

La universidad actual enfrenta grandes retos para "insertarse en un mundo complejo, con nuevas exigencias del mercado y competencias emergentes asociadas a la sociedad del conocimiento" (González, 2008, p.248). Una conclusión inicial radica en afirmar que la educación de calidad es el motor del desarrollo, que contribuirá en la medida que se mejoren los sistemas educativos a resolver los principales problemas mencionados en el discurso del desarrollo.
"La educación y el conocimiento son parte sustancial para alcanzar un nuevo desarrollo" (Didriksson, 2000, p.11). En este entendido, el gran reto de las universidades en la actualidad es comprender a que se refiere una educación de calidad, ya que existen muchas interpretaciones al respecto, y es necesario primeramente asumir las implicaciones de este concepto que va más allá de una acreditación y evaluación. Por un lado, puede decirse que la educación de calidad debe ser vista como la mejora de la educación que responda a las necesidades de la sociedad, por otro lado, los gobiernos de los países deben apostar en la inversión de capital humano y financiero con el objetivo de mejorar la educación, y de esta manera potencializar un nuevo desarrollo.

Dentro del discurso del desarrollo siempre ha estado presente la educación como detonante del mismo. Incluso desde la década de 1980 surge el paradigma de la educación de calidad como respuesta a la política educativa, consolidándose posteriormente con el surgimiento de organismos acreditadores de la educación superior. En tanto, la literatura acerca del discurso del desarrollo (teorías del desarrollo), posee una estrecha relación con el tema de la educación, ya que esta prepara a las personas para el ejercicio ciudadano y profesional, reduce la desigualdad y pauperización.

La educación de calidad en la actualidad se convirtió en un objetivo global a partir de los ODM establecidos por las Naciones Unidas en el 2000. Pese al fracaso de dichos objetivos en el 2015 se 
establecieron los ODS de la agenda 2030, en los cuales se plantea la educación de calidad como un objetivo que potencializa el desarrollo en los países menos avanzados. En concreto, la educación de calidad a partir de los ODS es imperante, ya que la influencia de organismos internacionales hace un llamado a los gobiernos de los países a mejorar la educación en todos los niveles. En efecto, la adopción de una cultura de educación de calidad coadyuvará a potencializar el desarrollo en los países.

Por lo que respecta a la educación superior, considerando este nivel clave para el desarrollo integral de un país, es relevante considerar que los procesos de acreditación han jugado un papel protagónico al hablar de educación de calidad. Sin embargo, es necesario centrar la atención no solo en la parte administrativa sino en la operativa; en el proceso de enseñanza, vinculación, investigación y formación integral que capacite al estudiante para el ejercicio ciudadano y profesional.

Así, el reflexionar sobre la educación de calidad en la educación superior a partir del discurso del desarrollo permite identificar la relación que posee este tema con otros problemas colaterales del desarrollo. En tanto, se recomienda que las IES interpreten la educación de calidad como la mejora de todos los procesos educativos que implican el servicio educativo.
Derivado de la reflexión establecida en este documento, es imprescindible retomar que las teorías del desarrollo y la educación poseen un vínculo importante, ya que ambas persiguen contribuir al desarrollo de un país. Por su parte, la acreditación juega un rol protagónico por alcanzar los fines mismo del discurso del desarrollo, sin embargo, es necesario profundizar en función de las limitantes que este proceso ocasiona en las instituciones educativas.

\section{REFERENCIAS}

Acosta-Ochoa, A. (2014). Evaluación y acreditación de programas educativos en México: revisar los discursos, valorar lo efectos. ANUIES, 43(4), 151-57. Obtenido de

http://www.scielo.org.mx/scielo.php?script=s ci arttext\&pid=S0185-27602014000400009

Arnove, R. (2006). La educación en América Latina: dependencia, subdesarrollo y desigualdad. Revista UAB, pp. 47-66. Obtenido de https://ddd.uab.cat/pub/caplli/2006/174868/e duamelat a2006.pdf

Avendaño-Castro, W., Paz-Montes, L. S., \& Parada-Trujillo, A. (2016). Estudio de los factores de calidad educativa en diferentes instituciones educativas de Cucuta. Investigación \& Desarrollo, 24(2), 329-354. Obtenido de https://www.redalyc.org/pdf/268/2685008600 6.pdf

Bedoya-Abella, C. L. (2011). Amartya Sen y el desarrollo humano. Revista Memorias.indb, pp. 277-288. Obtenido de https://d1watxts1xzle7.cloudfront.net/567373 91/desarrollo humano amartya sen.pdf?15 28267131=\&response-contentdisposition=inline $\% 3 \mathrm{~B}+$ filename $\% 3 \mathrm{DD}$ Desarro llo humano amartya sen.pdf\&Expires $=1595$ 288017\&Signature=YV5RvWuV3NOCZfxxZ YNDaACB-Kd7GEixqFzFp20AP7yWrg1oD 
Bernasconi, A., \& Rodríguez, E. (2018).

Importancia de la gestión institucional en los procesos de acreditación universitaria en Chile. Revista de Ciencias Humanas y Sociales (86), 20-48. Obtenido de https://dialnet.unirioja.es/servlet/articulo?codi go=7338167

Cuéllar-Saavedra, Ó., \& Moreno-Armella, F. (2009). Del crecimiento económico al desarrollo humano. Los cambiantes usos del concepto de desarrollo en América Latina, 1950-2000. Revista Sociológica, 24(70), pp.83-114. Obtenido de http://www.scielo.org.mx/scielo.php?script=s ci arttext\&pid=S0187-01732009000200004

Didriksson, A. (2000). La Universidad del futuro. Relaciones entre la educación superior, la ciencia y tecnología. México, D.F.: CESU.

Egidio-Galvez, I., \& Haug, G. (2006). La acreditación como mecanismo de garantía de la calidad: tendencias en el Espacio Europeo de Educación Superior. Revista Española de Educación Comparada (12), pp. 81-112. Obtenido de http://revistas.uned.es/index.php/REEC/articl e/view/7425/7093

Escobar, A. (2007). La invención del Tercer Mundo. Construcción y deconstrucción del desarrollo. Caracas, Venezuela: Fundación Editorial el perro y la rana.

Esteva, G. (1996). Diccionario del desarrollo. Una guía del conocimiento como poder. Perú: PRATEC.

González, L. E. (2008). Calidad de la educación superior: conceptos y modelos. Revista Calidad en la Educación (28), pp. 248-260. doi:http://dx.doi.org/10.31619/caledu.n28.21 $\underline{0}$

González-Pérez, I. (2011). Desarrollo y cultura tras los Objetivos del Milenio: consecuencia en la cooperación educativa internacional. Revista Española de Educación Comparada, 17, pp. 31-64. Obtenido de http://revistas.uned.es/index.php/REEC/articl e/view/7544
Hernández-Mondragón, A. R. (2006). La acreditación y certificación en las instituciones de educación superior. Hacia la conformación de circuitos académicos de calidad: ¿Exclusión o Integración? Revista del Centro de Investigación, 7(28), pp. 5161. Obtenido de https://www.redalyc.org/pdf/342/34202604.p $\underline{\mathrm{df}}$

Hernández-Mondragón, A. R. (2006). La acreditación y certificación en las instituciones de educación superior. Hacia la conformación de circuitos académicos de calidad: ¿exclusión o integración? Revista del Centro de Investigación, 7(26), pp. 5161. Obtenido de https://www.redalyc.org/pdf/342/34202604.p $\underline{\mathrm{df}}$

Lemaitre, M., Maturana, M., Zenteno, E., \& Alvarado, A. (2012). Cambios en la gestión institucional en universidades, a partir de la implementación del sistema nacional de aseguramiento de la calidad: la experiencia chilena. Revista Calidad en la Educación, 36, 21-52. Obtenido de https://scielo.conicyt.cl/scielo.php?script=sci arttext\&pid=S0718-45652012000100001

López-Noriega, M. D., Lagunes-Huerta, C. A., \& Recio-Urdaneta, C. E. (2009). Políticas públicas y educación superior en México. $X$ Congreso Nacional de Investigación Educativa, pp. 1-14. Obtenido de https://www.comie.org.mx/congreso/memori aelectronica/v10/pdf/area tematica 09/pone ncias/1480-F.pdf

López-Portillo, V. (2019). Educación Superior de Calidad y Acreditación en México. El caso del Consejo para la Acreditación de la Educación Superior (COPAES). Revista Educación Superior y Sociedad, 22(22), p. 65-84. Obtenido de http://www.iesalc.unesco.org/ess/index.php/ ess3/article/view/33/33 
Moreno-Brid, J. C., \& Ruíz-Napóles, P. (2010). La educación superior y el desarrollo económico en América Latina. Revista Iberoamericana de Educación Superior, 106. Obtenido de https://repositorio.cepal.org/bitstream/handle 111362/4884/S2009334 es.pdf

Munive-Villanueva, M. Á. (2007). La acreditación: ¿mejora de la educación superior o atractivo artilugio estético? Revista en Enseñanza e Investigación en Psicología, 12(2), pp. 397408. Obtenido de https://www.redalyc.org/pdf/292/29212213.p df

Organización de las Naciones Unidas. (2015). Objetivos de Desarrollo Sostenible. Obtenido de Agenda 2030:

http://www.onu.org.mx/agenda2030/objetivos-del-desarrollo-sostenible/

Organización de las Naciones Unidas para la Educación. (2019). Educación 2030. Obtenido de Desglosar el Objetivo de Desarrollo Sostenible 4: https://www.buenosaires.iiep.unesco.org/site s/default/files/archivos/ODS4_0.pdf

Oriol-Prats, J. (2006). Teoría y práctica del desarrollo. Cambios en las variables de la "ecuación del desarrollo" en los últimos 50 años. Revista del CLAD Reforma y Democracia (36), pp.1-17. Obtenido de https://www.redalyc.org/pdf/3575/357533668 003.pdf

Orozco-Cruz, J. C., Olaya-Toro, A., \& VillateDuarte, V. (2009). ¿calidad de la educación o educación de calidad? una preocupación más allá del Mercado. Revista Iberoamericana de Educación (51), pp. 161181. Obtenido de https://rieoei.org/historico/documentos/rie51a $\underline{08 . p d f}$

Pérez-Nájera, J. A. (2020). La desigualdad del ingreso en México. Una aproximación desde las teorías del desarrollo. Revista Interconectando Saberes (I.S.), 5(9), pp. 187-198. Obtenido de http://is.uv.mx/index.php/IS/article/viewFile/2 $\underline{655 / 4561}$
Programa de las Naciones Unidas para el Desarrollo. (2015). Objetivos de Desarrollo del Milenio. Obtenido de Informe 2015: http://mdgs.un.org/unsd/mdg/Resources/Stat ic/Products/Progress2015/Spanish2015.pdf

Reyes, G. (2001). Revista Crítica de Ciencias Sociales y Jurídicas. Principales teorías sobre el desarrollo económico y social (4), pp. 1-23. Obtenido de https://www.redalyc.org/pdf/181/18100408.p $\underline{\mathrm{df}}$

Rivas, P. (2014). Educación, calidad y globalización. Revista ONTOSEMIÓTICA, 1(1), pp. 9-18. Obtenido de http://erevistas.saber.ula.ve/index.php/ontos emiotica/article/view/5347

Rodríguez-Pérez, I. (2007). La acreditación de los programas educativos, ¿eleva la calidad de la educación? Revista Iberoamericana de Producción Académica y Gestión Educativa, 1(2). Obtenido de https://pag.org.mx/index.php/PAG/article/vie $\underline{w / 198}$

Silas-Casillas, J. C. (2014). Calidad y Acreditación en la Educación Superior: realidades y retos para América Latina. Revista Educación, 7(2), pp. 104-123. Obtenido de http://www.scielo.edu.uy/scielo.php?script=s ci arttext\&pid=S1688$\underline{74682014000200006 \& \operatorname{lng}=e s \& t \operatorname{lng}=e s}$

Tellería, J. (2015). ¿Seguimos hablando de desarrollo? El paradigma del desarrollo humano del PNUD como saber-poder. Revista Nómadas (43), pp. 241-51. doi:10.30578/nomadas.n43a14

Tünnermann-Bernheim, C. (2003). La universidad latinoamericana ante los retos del siglo XXI. México: UDAL.

Tünnermann-Bernheim, C. (2008). La calidad de la Educación Superior y su Acreditación: la experiencia centroamericana. Revista Avaliação, 13(2), pp. 313-336. Obtenido de https://www.scielo.br/scielo.php?script=sci a $\underline{\text { rttext\&pid=S1414-40772008000200005 }}$ 
Villalobos-Monroy, G., \& Pedroza-Flores, R.

(2009). Perspectiva de la teoría del capital humano acerca de la relación entre educación y desarrollo económico. Revista tiempo de educar, 10(20), pp. 273-306.

Obtenido de

https://www.redalyc.org/pdf/311/3111298700

2.pdf 